\title{
Towards Bridging the Gap Between BDA Challenges and BDA Capability: A Conceptual Synthesis Based on a Systematic Literature Review
}

\author{
Nico Hirschlein \\ University of Bamberg, Germany \\ nico.hirschlein@uni-bamberg.de
}

\author{
Jan-Niklas Meckenstock \\ University of Bamberg, Germany \\ jan-niklas.meckenstock@uni- \\ bamberg.de
}

\author{
Christian Dremel \\ Norwegian University of Science \\ and Technology, Norway \\ christian.dremel@ntnu.no
}

\begin{abstract}
Big data analytics (BDA) and strategies for implementing $B D A$ have received attention among researchers and practitioners alike. However, success stories pertaining to the implementation of BDA remain scarce. The notion of the BDA deployment gap describes the chasm between the attributed value potential of $B D A$ and its actual value realization in organizational practice. Several research articles indicate challenges encountered in implementing BDA but lack a comprehensive systematization of BDA implementationrelated challenges. This research article aims to systematize those challenges through a systematic literature review. As a result, we derived five overarching challenge dimensions related to the $B D A$ implementation. Based on this systematization, we adopt the lens of a big data analytics capability and delineate future research avenues through the derivation of propositions on how to overcome the BDA implementation-related challenges, while enhancing our understanding about how to solve the BDA deployment gap.
\end{abstract}

\section{Introduction}

Big data (BD) epitomizes the enormous potential to enable data-driven decision-making and is seen as the new oil for organizations [1], embodying the next management revolution [2]. However, only the effective analysis and use of BD, called big data analytics (BDA), unfolds the exhaustive potential for business value creation in organizations, facilitating the path from insights to value [2]. BDA refers to the technologies, techniques, and processes for using $\mathrm{BD}$ to create and realize business value. For instance, the creation and realization of business value targets output metrics like productivity gains and revenue growth [3]. Nonetheless, the business value realization requires the establishment of contingent technical assets and complementary resources [1].

During the past decade, many organizations tried to adopt and implement BDA, as BDA is nowadays seen as a necessary technical artifact to stay competitive within an organization's environment [2]. Though many organizations try to adopt and implement BDA, successful implementation stories remain scarce within IS research [4]. The notion of the BDA deployment gap depicts this chasm, stating that there is a significant discrepancy between the perceived business value potential of BDA and its actual value realization and implementation success within organizations $[4,5,6]$. The underlying theoretical lens for explaining value realization mechanisms from BDA is embodied through the resource-based view (RBV) and the concept of capabilities [1]. This lens delineates the process behind value creation and realization by explaining the required BDA-related resources and capabilities [7]. Extant research identified the constitutive elements of a big data analytics capability and studied its effects on output variables like firm performance and business value realization [1,7], using a set of theoretical perspectives like the RBV, contingency theory, and service-dominant logic $[8,9,10]$. As a complementary aspect, several articles studied BDA adoption with the goal to identify critical success factors and adoption challenges $[5,11]$. However, there are two key shortcomings within the extant body of literature. First, capability-based perspectives on BDA only focus on resource-picking aspects and explain what resources are required for realizing value, while neglecting to answer how to orchestrate these resources [12]. Second, there is a lack in identifying recommendations for the implementation of BDA and how to address the BDA deployment gap, as previous research only focuses on the enumeration of key implementation challenges $[4,5]$. To address these shortcomings, we propose the following research question (RQ): How can BDA-specific implementation challenges be systematized and overcome through the establishment of BDA-related resources? 
To answer our RQ, we rely on a systematic literature review. The next section depicts the theoretical background for our research endeavor and outlines the implementation and capability-based perspective on BDA. In the subsequent sections, we propose a systematization of BDA implementationrelated challenges and conclude our article with first steps towards a mapping of challenges to resources, proposing fruitful avenues for future research.

\section{Theoretical foundations}

\subsection{Big data \& big data analytics in IS research}

Big data (BD) represents one of the most prominent buzzwords in IS research for more than 10 years $[1,13]$. The hype around BD is particularly due to its promised potential for business value realization [3]. Both researchers and practitioners agree on defining BD based on five distinct characteristics, the so-called V's. For the context of our research endeavor in overcoming the BDA deployment gap, we comprehend BD along the attributes volume, variety, veracity, velocity, and the derivable value $[3,13,14,15,16]$. From our viewpoint, the effective use of $\mathrm{BD}$ refers to the notion of big data analytics (BDA), which embodies the key technical artifact for our article [1]. Within IS research, BDA is defined through different perspectives. Sample definitions specify BDA as lifecycle and concept for analyzing and interpreting data $[9,16]$, or as application of analytical techniques to advance business [17]. Some articles have proven the value potential of BDA, e.g., through the establishment of a BDA infrastructure [17], or the orchestration of contingent resources [8]. Hence, BDA is called to drive value creation. Consequently, we define BDA as technologies, techniques, and processes for using $\mathrm{BD}$ to realize business value.

\subsection{Big data analytics deployment gap \& implementation-related challenges}

Organizations willing to implement BDA as a means of value creation encounter a diverse series of potential challenges during the implementation process. A phenomenon pertaining to these challenges, which was frequently observed in the extant body of literature, is depicted as the BDA deployment gap $[5,6]$. This term relates to the "paradox between the enormous potential of BD across industries, on one hand, and the observation that actual deployments of BD business models remain scarce, on the other hand" [4]. The scarcity of BDA business models is due to the fact that those require a successful BDA implementation.
Reasons for the existence of the described gap and the associated rarity of successful implementations prevalently relate to the challenges encountered in the implementation process [5]. Deployment gaps and lag effects are commonly encountered phenomena in IS research. However, the discrepancy between the assumed value potential of BDA and its actual value realization in practice is significantly more prominent compared to other information technologies. The preliminary perception gained in initial investigations on implementations of BDA is that anchoring BDA in a firm poses BDA-specific technical, organizational, and personnel-related challenges [11]. From a theoretical standpoint, it thus far remained unclear, how to overcome the challenges that impede a successful BDA implementation. These challenges will therefore be dissected in detail in chapter 4 . What is even more salient, however, is the observed heterogeneity of terms used to delineate the challenges encountered in the implementation process. Exemplary notions include obstacles, barriers, issues, impediments, and roadblocks, while the expressions all pertain to the same concept that hampers the implementation of BDA [18, $19,20,21]$. To establish a common understanding of the challenges that firms are required to overcome for a successful BDA implementation, we conceptualize the term "BDA implementation-related challenges". This term covers the entire breadth of expressions identified in the extant body of literature, that potentially impede the effective BDA implementation in organizations.

\subsection{A capability-lens on big data analytics using the resource-based view of IT}

The resource-based view (RBV) represents the most renowned theoretical paradigm to explain possibilities of organizational value creation and realization [1, 22]. In line with the extant body of knowledge, the concepts of resources and capabilities out of the RBV are prevailing in explaining mechanisms for value realization from $\operatorname{BDA}[1,7,9,17]$. Thus, the process of BDA value realization relies on contingent resources and capabilities [8, 23]. Taking a capabilityoriented stance, the term of a big data analytics capability (BDAC) has proven as theoretical driver in explaining the mechanisms behind organizational benefits through the usage of BDA $[1,7]$.

There is consensus in the IS research community to define a BDAC through the lens of the RBV along its constituent elements, incorporating technical, human, and intangible resources [1,9]. Through this lens, several articles provide exhaustive insights for each superordinate BDA-related resource [7, 9, 23, 24, 25].

Pertaining to the technical resource category, existing articles emphasize the need for establishing a multi- 
layered BDA infrastructure with several characteristics like modularity and flexibility, and a corresponding management for processing and analyzing data [7, 9]. Human resources refer to the whole necessary skill-set at the employee-level to derive insights out of $\mathrm{BD}$, summarized with the notion of data literacy [26]. Intangible resources tackle all required complementary resources to handle BDA in organizations, including governance, structures, and culture $[1,7]$. In the light of our research endeavor, we adapt and use the distinct elements of a BDAC to explain what BDA-related resources are required to overcome certain BDA implementation-related challenges. In line with the extant body of knowledge, we argue for orchestrating BDA-related resources into a BDAC to entirely surmount those challenges and effectively implement BDA within organizations [12].

In summary, we define a big data analytics capability as the organizational competence of deploying and orchestrating BDA-related resources, that enable an organization to solve the BDA deployment gap. A successful overcoming of the different challenges requires the existence of BDArelated resources congruent to the challenges, which need to be synchronized and integrated in an organizational BDAC.

\section{Research methodology}

To answer the RQ posed in the introductory section, we conducted a systematic literature review to summarize the current state of research on BDA implementations in organizations. In addition, we pursue the objective of bridging the deployment gap by depicting BDA implementation-related challenges and possible solution avenues. In doing so, we followed the guidelines for a systematic literature analysis proposed by vom Brocke et al. (2009) [27].

As a starting point, we substantiated the research focus in defining the central terms and underlying concepts that refer to BDA implementation-related challenges and the foundations and elements of a BDAC. This step entails the elaboration and definition of the term "BDA implementation-related challenges". Correspondingly, the literature analysis pertaining to the capability lens comprises the identification of the key elements of a BDAC, especially regarding the individual potential of BDA-related resources required to overcome the previously identified BDA implementation-related challenges. Former research has thematized BDA implementation-related challenges to some extent, while the bandwidth of implementationrelated challenges remains rather limited. Initial attempts within the extant body of knowledge are therefore already discussed in chapter 2 .
The literature search was streamlined around our proposed research question and focused on BDA implementation-related challenges and BDA-related resources and capabilities. The employed search strings represented systematic combinations of terms pertaining to the two overarching topics, namely "big data analytics", and synonyms of the term "challenges" such as "barriers" and "obstacles", as well as "capability" and "resource" for the BDAC section.

We scanned the most prominent databases for IS research (AISel, IEEE Xplore, ACM digital library, Science Direct, EBSCO Host, T\&F) using our search strings, focusing on results from the last 10 years. For the identification of relevant articles, we employed an abstract-based screening method and applied inclusion and exclusion criteria to evaluate the relevance of an article for our review. Hence, we only included articles that specifically discuss BDA-related challenges and excluded items that only slightly touched the focal topic. Subsequently, we assessed the quality of articles along two distinct rankings, namely the VHB Jourqual 3 ranking and the journal ranking developed by the Australian Business Dean Council 2019. To substantiate our findings, we frequently discussed the individual relevance of articles within our research group. Our literature search led to 20 hits in the BDA implementation-related challenges domain and 12 hits on the concept of BDAC.

Following to the completed literature search, we analyzed the 20 identified articles on BDA implementation-related challenges using a systematic coding procedure. Hence, we adopted a three staged coding procedure along the steps of open, axial, and selective coding, suggested by Gioia et al. (2013) and Corbin \& Strauss (1998) [28, 29]. In the first step of our coding procedure, we extracted text fragments from the articles in our review sample and coded them separately. This resulted in 218 single challenge statements. During the axial coding step, we aggregated the single challenges into $152^{\text {nd }}$ order themes, which were lastly summarized into five dimensions of implementationrelated challenges. The whole coding and mapping process was conducted in a collaborative manner, which included iterative discussions between the three authors.

Concluding our research endeavor, we aim to provide first theoretical and empirical insights on how to overcome BDA implementation-related challenges through BDA-related resources. Therefore, our identified $2^{\text {nd }}$ order themes serve as starting point for our mapping. For each challenge dimension, we selected one theme that appeared most pertinent within our identified review articles, based on its frequency within the extant body of literature (see Table 1). Subsequently, the selected $2^{\text {nd }}$ order themes were analyzed through a BDAC lens. The identification of BDA-related 
resources was conducted through the usage of the extant body of knowledge in form of our review articles on the constitutive elements of a BDAC. Building on the selected adequate BDA-related resource, the resource is explained in the light of the challenges and substantiated with concrete action items on how to overcome them. The deduction of concrete action items was performed through the analysis of case studies that thematized BDA implementations. We identified eight case studies that provide in-depth insights on how to implement BDA within an organization $[6,30,31,32,33,34,35$, 36] in a systematic literature review using the above mentioned search terms in combination with the term "case study", using the same databases. Based on the application of the capability-lens and the addition of indepth case study insights, we derived propositions for future research endeavors on how to overcome the BDA deployment gap.

Table 1. Systematization of BDA implementation-related challenges ( ${ }^{*}$ selected theme f. mapping)

\begin{tabular}{|c|c|c|c|c|}
\hline Dimension & Description of Dimension & $2^{\text {nd }}$ order theme & References & $\Sigma$ \\
\hline \multirow{3}{*}{$\begin{array}{c}\text { Infrastructure \& } \\
\text { Technology-related } \\
\text { challenges }\end{array}$} & \multirow{3}{*}{$\begin{array}{l}\text { Challenges affecting the } \\
\text { overall BDA infrastructure } \\
\text { and toolset, the individual } \\
\text { technical layers, and their } \\
\text { joint integration }\end{array}$} & $\begin{array}{l}\text { Missing / immature / inadequate } \\
\text { BDA infrastructure for the } \\
\text { proper handling of data }\left(^{*}\right)\end{array}$ & $\begin{array}{l}\text { [2], [11], [18], [21], [37], } \\
\text { [38], [39], [40], [41], [42], } \\
\text { [43], [44], [45] }\end{array}$ & 13 \\
\hline & & $\begin{array}{l}\text { Fragmented IT landscape and } \\
\text { technology architecture }\end{array}$ & $\begin{array}{l}\text { [2], [11], [18], [19], [38], } \\
{[41],[42],[44],[45],[48]}\end{array}$ & 10 \\
\hline & & $\begin{array}{l}\text { Issues with BDA-related tools } \\
\text { and applications }\end{array}$ & [2], [38], [40], [43] & 4 \\
\hline \multirow{3}{*}{$\begin{array}{c}\text { Data \& Data } \\
\text { Management related } \\
\text { challenges }\end{array}$} & \multirow{3}{*}{$\begin{array}{l}\text { Challenges occurring along } \\
\text { the entire analytical lifecycle } \\
\text { that affect the handling and } \\
\text { usage of an organization's } \\
\text { big data from the initial data } \\
\text { collection to the creation of } \\
\text { actionable insights }\end{array}$} & $\begin{array}{l}\text { Persistent issues of data usage } \\
\text { and handling over the whole } \\
\text { analytics lifecycle }\left(^{\star}\right)\end{array}$ & $\begin{array}{l}\text { [11], [18], [19], [20], [21], } \\
{[37],[38],[39],[40],[43],} \\
{[45],[46],[47],[49]}\end{array}$ & 14 \\
\hline & & $\begin{array}{l}\text { Data quality issues preventing } \\
\text { further processing and business } \\
\text { value creation }\end{array}$ & $\begin{array}{l}\text { [11], [18], [19], [21], [37], } \\
{[38],[39],[40],[43],[45],} \\
{[46],[47],[49]}\end{array}$ & 13 \\
\hline & & $\begin{array}{l}\text { Lack of adequate analytical } \\
\text { procedures to analyze data }\end{array}$ & $\begin{array}{l}\text { [18], [19], [37], [38], [42], } \\
{[45],[46],[47]}\end{array}$ & 8 \\
\hline \multirow{3}{*}{$\begin{array}{l}\text { Skill and Expertise } \\
\text { related challenges }\end{array}$} & \multirow{3}{*}{$\begin{array}{l}\text { Challenges emerging from } \\
\text { the lack of BDA } \\
\text { competencies at the } \\
\text { employee-level and the } \\
\text { corresponding inadequate } \\
\text { talent management in the } \\
\text { organization }\end{array}$} & Missing data literacy $\left({ }^{*}\right)$ & $\begin{array}{l}\text { [2], [11], [18], [21], [38], } \\
{[39],[40],[43],[44],[45],} \\
{[46]}\end{array}$ & 11 \\
\hline & & $\begin{array}{l}\text { Unfocused and fragmented } \\
\text { talent management, acquisition, } \\
\text { and retention }\end{array}$ & $\begin{array}{l}\text { [2], [11], [19], [41], [43], } \\
{[44],[45],[46],[47]}\end{array}$ & 9 \\
\hline & & $\begin{array}{l}\text { Lack of adequate education } \\
\text { programs to overcome skill } \\
\text { shortages }\end{array}$ & $\begin{array}{l}\text { [11], [38], [40], [43], [48], } \\
{[49]}\end{array}$ & 6 \\
\hline \multirow{3}{*}{$\begin{array}{c}\text { Organization \& } \\
\text { Management related } \\
\text { challenges }\end{array}$} & \multirow{3}{*}{$\begin{array}{l}\text { Challenges arising from } \\
\text { inadequate organizational } \\
\text { structures and management } \\
\text { processes that steer the } \\
\text { BDA implementation efforts }\end{array}$} & $\begin{array}{l}\text { Lack of top-management } \\
\text { guidance and investment (*) }\end{array}$ & $\begin{array}{l}\text { [11], [13], [18], [19], [20], } \\
{[38],[40],[41],[43],[44],} \\
{[46],[47],[48],[49]}\end{array}$ & 14 \\
\hline & & $\begin{array}{l}\text { Inadequate organizational } \\
\text { frame, and working modes }\end{array}$ & $\begin{array}{l}\text { [11], [13], [18], [38], [41], } \\
\text { [42], [46], [48], [49] }\end{array}$ & 9 \\
\hline & & $\begin{array}{l}\text { Lack of a unified governance } \\
\text { framework for BDA }\end{array}$ & $\begin{array}{l}\text { [2], [11], [13], [18], [38], } \\
{[39],[47],[49]}\end{array}$ & 8 \\
\hline \multirow{3}{*}{$\begin{array}{l}\text { Culture-related } \\
\text { challenges }\end{array}$} & \multirow{3}{*}{$\begin{array}{l}\text { Challenges pertaining to the } \\
\text { understanding, attitude, and } \\
\text { mindset towards BDA and } \\
\text { its impact on transforming } \\
\text { the whole organization }\end{array}$} & $\begin{array}{l}\text { Lack of business-IT alignment } \\
\text { due to missing collaboration } \\
\text { between business and IT (*) }\end{array}$ & $\begin{array}{l}\text { [11], [13], [18], [20], [21], } \\
\text { [42], [44], [46], [48], [49] }\end{array}$ & 10 \\
\hline & & $\begin{array}{l}\text { Missing data-driven mindset } \\
\text { and shared corporate } \\
\text { understanding about BDA }\end{array}$ & $\begin{array}{l}\text { [11], [18], [38], [41], [42], } \\
{[43],[47],[48]}\end{array}$ & 8 \\
\hline & & $\begin{array}{l}\text { Resistance to change towards } \\
\text { acceptance of BDA }\end{array}$ & $\begin{array}{l}\text { [11], [18], [21], [41], [44], } \\
\text { [46], [47] }\end{array}$ & 7 \\
\hline
\end{tabular}

\section{A systematization of BDA implementation-related challenges}

The results of our coding analysis are explained in Table 1. We identified five distinct dimensions of BDA implementation-related challenges as well as three summarized themes that further detail each identified dimension. Each dimension is further described below.

Infrastructure- and technology-related challenges. This dimension includes challenges referring to the overall BDA infrastructure, the single layers in the BDA stack, and the integration of BDAspecific tools within the technology infrastructure. It was notably apparent that an immature and inadequate BDA infrastructure causes also various challenges in other areas like data management. These include problems with the bandwidth required for instant data transmission allowing for real-time processing [37, 38, 
39], and a lack of scalability and integration of data storage units for large datasets [18, 19, 37, 38, 39, 40]. As a whole, BDA requires a powerful infrastructure that enables an organization to gain insights from the available datasets and extract value through the application of data analysis [2, 19, 41, 42, 43]. The establishment of a unified IT architecture is closely related to the described immature BDA infrastructure characteristics. In particular, a fragmented IT architecture reduces the interoperability between corporate IT systems and the BDA technology stack, requiring the establishment and validation of system connectivity [11, 18, 41, 42, 44, 45]. Moreover, the lack of available BDA-specific tools can diminish the functionality of the BDA technology stack [38, 43].

Data- and data management-related challenges. This dimension concerns issues that can be attributed to the data itself and the associated data processing and analysis. A frequently observed phenomenon is the insufficient data quality, recognizable through a lack of data standardization, a high degree of data heterogeneity, and data inconsistencies as well as incompleteness $[18,19,21,37,38,43,44,46]$. Observable consequences of insufficient data quality encompass interpretability, reliability issues as well as lower trustworthiness of derived insights $[11,37,39,40$, $45,47]$. The unique characteristics of BD furthermore affect the utilization of data along the entire analytical lifecycle. Exemplary challenges within this lifecycle include data transmission, data integration and (pre-) processing, data mining and analysis, data modeling, and data accessibility $[19,20,37,38,39,40,43,45,46$, 47]. Further issues arise from the inherent security and privacy concerns associated with $\mathrm{BD}[18,19,21,37,38$, $40,42,43,47]$. The deficiencies of analytical techniques, which can be applied to different datasets, are closely related to the above-mentioned lack of BDA tools. Both issues hamper the derivation of actionable insights from the analyzed datasets [18, 19, 37, 42, 45].

Skill- and expertise-related challenges. The third identified dimension delineates challenges related to skills and expertise on the employee-level, especially regarding the creation, development, and management of BDA-related competencies. Firms require a focused talent management to hire and retain skilled BDA personnel, including data scientists and engineers. However, many organizations struggle in creating a focused talent management competency, resulting in a shortage of well-trained employees to support a successful BDA endeavor [11, 41, 43, 44, 45, 46, 47]. The described scarcity of skilled BDA-experts in the organization is further aggravated by the current shortage of specialists on the labor market $[11,19,40$, $42,44,46]$. The resulting lack of data literacy poses further challenges. This includes a lack of technical, analytical, managerial, and relational skills $[11,13,18$, 20, 21, 38, 44, 46, 47, 48]. As described by Vidgen et al. (2017) and Dremel (2017), the establishment of domain knowledge can therefore be seen as a key success factor for BDA [47, 48]. Thus, it is necessary to create an integrated BDA competence spanning across technical and managerial domains, which requires the formation of a central education program. However, dedicated training programs to educate staff on BDA are yet rarely established in organizations [11, 38, 40, 43, 48, 49].

Organization- and management-related challenges. This dimension particularly describes challenges referring to a strategic management of the BDA implementation at the organizational level. The challenges belonging to this dimension accentuate the crucial role of top management, including funding, strategic vision, and commitment towards the BDAdriven transformation $[11,13,18,19,38,41,43,44,46$, 47]. Top management is required to define, measure, and control the business value realizable through the implementation of BDA, thus to justify the business case and the corresponding investments $[11,18,40,47$, 48, 49]. Besides top management support, an organization requires the introduction of an efficient governance framework to control and structure BDA initiatives across the organization. However, standardized approaches to govern BDA in organizations are not available yet, which makes it difficult for organizations to effectively govern the entire BDA implementation process. This includes roles, accountabilities, and consistent processes [11, 38, 39, 47, 49]. The specification of appropriate organizational structures for BDA projects is inherently interwoven with governance of BDA efforts. Hence, BDA requires an organizational frame, including adapted collaborative structures and working processes [38, 41, 42, 46, 48, 49]. These structures need to be accompanied by agile project management and software development methods to support a swift adaptation in turbulent environments with a high degree of uncertainty [42, 46, 48].

Culture-related challenges. This dimension focuses on issues regarding the behavioral and general attitude towards BDA. Thereby, the core of those challenges concerns missing business IT alignment, which is required for the execution of successful BDA projects $[11,13,18,20,21,42,44,46,48,49]$. A tight collaboration and mutual understanding between business and IT experts is necessary to secure a business acumen within the BDA projects [48]. The realization of business IT alignment entails a mindset change pertaining to the acceptance of BDA and its effects on the organization. While business IT alignment can also be viewed through a structural lens, we considered the cultural notion of business IT alignment to be 
particularly important for the context of BDA implementation. These mindset changes and the corresponding cultural transformation are frequently impeded by a strong resistance to change $[11,18,21,41$, $44,46,47]$. This reluctance to change is due to a missing corporate understanding of what BDA effectively implies, thus lacking a deeply rooted anchoring of BDA appreciation within the corporate culture $[11,18,38,41$, $42,43,47,48]$. A lack of fact-based culture thus inhibits the effectiveness of data-driven decision-making [18].

In sum, we identified 5 overarching dimensions of BDA implementation-related challenges with 15 associated themes that further describe the characteristics of the classified challenges. The developed systematization summarizes the extant body of knowledge and serves as starting point for the subsequent mapping of challenges to the BDA-related resources of a BDAC required for a successful implementation of BDA.

\section{Discussion}

To derive initial recommendations to help overcome BDA implementation-related challenges that constitute the BDA deployment gap, we adopt a BDACoriented perspective. In the extant body of literature, establishing a BDAC is considered as an indispensable driver of implementation success and business value realization in organizations [1]. Departing from the BDAC and its constitutive elements, we aim at proposing a BDA-related resource that particularly addresses a certain BDA implementation-related challenge. Thereby, we substantiate the proposed resource with concrete action items inferred from the identified case studies to delineate how a specific pain point embodied in an identified challenge theme could be adequately treated. In doing so, it is important to acknowledge that overcoming the described challenges always entails the orchestration of multiple BDArelated resources from different categories, which reflects and emphasizes the capability-driven perspective on BDA [7]. To summarize the suggested efforts to overcome a certain challenge, we formulate propositions that capture the specific pain point and appropriate counteractions.

\subsection{A mapping of challenges and adequate BDA-related resources}

As a starting point, we focus on the challenges that are primarily addressed by technical resources. From our viewpoint, the challenges pertaining to infrastructure and technology as well as data and data management refer to this category. We adapt the BDAC perspective suggested by Gupta \& George (2016) [7], who assigned technology and data to the technical resource dimension. This allocation appears fitting, since the underlying infrastructure and the data that is managed based on this infrastructure along its lifecycle encompass the technical aspects of BDA.

Missing, immature, or inadequate BDA infrastructure. As part of the infrastructure- and technology-related challenges, this challenge pertains predominantly to the BDA infrastructure, its layers, and the interworking of these layers, aiming at ensuring a sufficient technical maturity level. To tackle this challenge, a BDA infrastructure and corresponding tools need to be gradually established [7]. As the findings in the cases indicate, the creation of a BDA infrastructure can be realized through different technical pathways. As described by Winig (2016) in the case of General Electrics (GE), a technical platform for connecting, storing, and analyzing data was created through the usage of a cloud-based solution called Predix [36]. The case study of Lufthansa reported by Chen et al. (2017) describes the creation of a serviceoriented architecture (SOA) for BDA [6]. The use of this type of architecture ensures modularity and flexibility in handling and integrating different system components and tools. In addition, Lufthansa uses an enterprise service bus as linkage between different IT systems, providing system interoperability [6]. Alternatively, the utilization of the Hadoop framework enables organizations to stepwise create a fully integrated BDA infrastructure, as reported by Dremel et al. (2020) [31]. To synthesize the different pathways to architecture realization in the cases, the establishment of a multilayered BDA architecture is recommended. As an initial starting point, a reference architecture suggested by Illa \& Padhi (2018) is used to illustrate the essential layers of a BDA architecture [50], addressing the tasks of data streaming and ingestion, data storage, data processing, and data visualization. To allow for maximum layer flexibility while ensuring structural cohesiveness, all layers within the BDA architecture must be connected to each other using predefined interfaces.

Data usage and handling over the analytics lifecycle. As part of data and data management-related challenges, many challenges in handling of $\mathrm{BD}$ arise, especially regarding data processing, storing, and interpreting, representing the whole analytics lifecycle. Renowned analytical lifecycles and process models including the popular CRISP-DM contain a diverse set of phases, ranging from business and data understanding to its deployment [51]. To tackle and overcome these issues, employees must be given the opportunity to experiment with $\mathrm{BD}$ to establish a collective sensemaking on how to use BD and its underlying infrastructure. The case study by Koch et al. (2021) 
reports on the necessary mindset to drive data handling and the establishment of data management processes [32]. According to Chen et al. (2017), data management processes should be accompanied by implementing several structural governance mechanisms within a data management framework, with the goal of establishing clear responsibilities for the data handling along the lifecycle [6]. As a starting point for the definition of data management processes, we suggest the consideration of the DAMA data management framework [52], which can be used to specify all necessary data management process domains. Based on the suggested framework, more concrete processes for the different domains like data security and metadata management can be derived.

Based on the insights on how to tackle the challenges regarding BDA architecture and data management, we derive the following proposition:

P1: To overcome challenges in the domain of technology and data management, the establishment of a multi-layered cohesive BDA infrastructure in orchestration with the instantiation of data management processes within a data governance framework is recommended.

Missing data literacy. As part of the skill- and expertise-related challenges, the notion of data literacy embodies an umbrella term for the required individual competences for handling and understanding BD [26]. BDAC involves human beings as a critical resource for successful BDA implementations. Human beings describe the essential resource required for effective sensemaking from the analysis of large datasets [23]. Effective sensemaking demands the proper usage of technical resources through a diverse skill set at the employee-level, while the management expertise needs to be aligned with the technical skills. However, many organizations report that their employees have an insufficient level of data literacy to drive BDA implementation efforts. The establishment of data literacy requires a central training and education program, as stated by Dremel et al. (2020): "We try to educate our employees and our top management [and] want to give them an understanding of the world of data at [PremiumCar]." [31] The benefits of an organizational education program arise from the central identification of training needs and the subsequent possibility of allocating adequate resources. Building on the notion of adequate resource allocation, the creation of a data-literate center of excellence $(\mathrm{CoE})$ supports the diffusion of a firm-wide BDA understanding, as stated by Krishnamoorthi \& Mathew (2018): "Then what is the role of the 500 people vis-a-vis the rest 99,500 people? I see our role as the incubator of framework and approaches to productize and commoditize Analytics" [33]. To tackle and overcome these issues, employees must establish sufficient knowledge and skills to be able to create actionable insights out of BDA. According to Mikalef et al. (2018) [1], employees need to be skilled within the technical, business, relational, and analytical domain. Firms need to be aware that all these skill domains are required for the creation of sufficient data literacy. Exemplary skills that belong to the data literacy concept include data engineering skills, business acumen, communication, and data visualization skills. These skills need to be developed in dedicated trainings.

Based on the insights on how to tackle the challenges regarding skills and expertise, we derive the following proposition:

P2: To overcome challenges in the domain of skills and expertise, the initial recognition of the required skillset for sufficient data literacy, which enables sensemaking through BDA, and the corresponding development of training programs is recommended.

The third constitutive element of a BDAC refers to intangible resources. From our viewpoint, intangible resources embody complementary organizational resources that particularly address challenges pertaining to organization \& management as well as corporate culture. Thereby, we follow the renowned IS business value perception of Melville et al. (2004) that conceptualizes an IT capability along technical IT resources, human IT resources, and complementary organizational resources [22].

Top management guidance and investment. As part of organization- and management-related challenges, both a lack in top management support as well as investments need to be overcome. The first step in establishing top-management support is the direct involvement of the C-level suite in BDA-related topics. An important aspect of top-management support is that funding and commitment need to be secured through a focused assessment of the potential business value derivable from the BDA implementation. Hence, a clear investment strategy needs to be developed by the topmanagement, which is driven by selected use cases that promise actual business value. The perspective of focused top-management support is detailed out of the CIO's perspective at Lufthansa: "However, we want to be with the leading technology but not the 'bleeding' technology. We are cautious. We do careful assessment of the big data technology" [6]. Top management involvement and value recognition need to be accompanied by a BDA governance framework to support a strategy-driven BDA implementation, as suggested by Chen et al. (2017): "We have a steering committee on the big data initiative; we went through our innovation process to discover value from big data, and we came up with a few lighthouse projects" [30]. Relying on the notions of Mikalef et al. (2020) [53], we suggest that a BDA governance framework needs to be developed along practices pertaining to structural, 
procedural, and relational dimensions. This framework needs to incorporate steering committees and a role taxonomy with defined responsibilities.

Lack of collaboration between business and IT experts. As part of culture-related challenges, key issues result from missing cross-departmental collaboration. The notion of business IT alignment is called to be a necessary pillar for the execution of successful BDA projects [9]. However, many organizations struggle in establishing a common ground for enabling a collaboration between business and IT employees. One way to achieve business IT alignment is the employment of an agile development method such as scrum, as outlined by Dremel et al. (2020): "We have to develop a flexibility and agility in regard to our releases. [...] Scrum is one possibility to achieve this and to get our product management and the developing team together" [31]. The introduction of new working modes that bring business and IT closer to another requires a change management process, as stated by Beath \& Ross (2010) in the case of PepsiAmerica: "For these initiatives to affect the entire organization or big pieces of it, you need to have a serious change management element to the project team. And that involves communication and education and training" [30]. Based on these recommendations, we formulate the following proposition:

P3: To overcome challenges in the domain of management and culture, the introduction of a BDA governance framework that includes novel interdisciplinary working modes, realized through a change management process, is recommended.

\subsection{Implications and limitations}

Our findings discussed in chapter 4 possess implications for academia and practice alike. The primary theoretical implication is embodied in a stateof-the-art systematization of the BDA implementationrelated challenges. We provide a structured overview of BDA implementation-related challenges, aiming at synthesizing the fragmented literature. Secondly, through an initial mapping of BDA-related resources to the identified BDA-implementation-related challenges, we propose a novel perspective on how to overcome these challenges using a BDAC lens.

The derivation of propositions out of this novel perspective informs practical BDA implementation endeavors in how to overcome the BDA deployment gap. Simultaneously, these propositions guide scholars in their future research endeavors, especially for the execution of qualitative studies, that make use of our proposed mapping of BDA-related resources to BDA implementation-related challenges. While prior attempts only viewed BDA implementation through a conceptual stance, the formulated propositions advance our understanding in how to overcome the BDA deployment gap through the explication of concrete action-oriented items. A possible future research avenue lies in measuring the impact of our identified BDArelated resources on certain output variables like implementation success and business value realization. Regarding implications for practitioners, our findings can help them select case-validated resources to tackle the challenges encountered in the BDA implementation on both strategic and operational levels. For the first time, practitioners are provided with more precise action items that have proven to help overcome previously identified challenges in real-case scenarios. Hence, our identified action items and corresponding resources serve as initial recommendations on how to successfully implement BDA within organizations.

Our findings have limitations that need to be considered when interpreting the results and possible implications. Most importantly, the extant of literature on BDA implementations lacks extensive coverage on successful cases from the industry. Therefore, the described action items originate from a limited number of analyzed case studies. In addition, the identified case studies merely superficially describe how BDA was implemented and rarely specify how to tackle encountered challenges. Secondly, this paper only takes organization-internal challenges into account, while potential external aspects that impede the BDA implementation were mostly neglected. Future research should thus also focus on external factors that pose challenges for a successful BDA implementation. Thirdly, we only proposed adequate BDA-related resources and propositions on one theme per challenge dimension due to page limitations. This holds true as well for the number of resources used for the mapping, which is also due to the limited variety of guiding action items in the identified case studies. Despite these limitations, our research still proposes avenues towards bridging the BDA deployment gap.

\section{Conclusion}

The underpinnings of a successful BDA implementation have for long depicted an opaque black box for both academia and practice. Previous research predominantly focused on necessary resources and capabilities that constitute a BDAC, whilst neglecting the need to address potential challenges encountered with dedicated resources to help overcome those challenges threatening a successful implementation. The visible result of this negligence constitutes the BDA deployment gap observed in practice. To close this gap, we conducted a systematic literature review on BDA implementation-related challenges and provide a 
structured systematization of challenges that occur during the whole implementation process of BDA. We synthesized the extant body of knowledge on BDA implementation-related challenges through the identification of five distinct challenge dimensions. Building on this systematization, we analyzed case studies pertaining to their BDA implementation efforts. Out of these in-depth insights, we developed an initial mapping of certain BDA implementation-related challenges to adequate BDA-related resources. Thereby, we build upon the extant body of literature on the constitutive elements of a BDAC. As a result, we formulated three propositions on how to overcome BDA implementation-related challenges. We intend to contribute to the body of knowledge on how organizations can successfully implement BDA and thus overcome the BDA deployment gap [4]. Based on our findings, we suggest future research to direct their endeavors in two possible directions. First, future research may empirically validate our propositions to gain a comprehensive understanding of overcoming the BDA deployment gap. A second direction points towards the practical investigation of additional BDA implementation cases to identify countermeasures that help overcome the BDA deployment gap. Solving these questions would help companies on their journey towards a data-driven organization.

\section{References}

[1] Mikalef, P., I.O. Pappas, J. Krogstie, and M. Giannakos, "Big data analytics capabilities: a systematic literature review and research agenda", Information Systems and eBusiness Management 16(3), 2018, pp. 547-578.

[2] Wamba, S., S. Akter, A. Edwards, G. Chopin, and D. Gnanzou, "How 'big data' can make big impact: Findings from a systematic review and a longitudinal case study", International Journal of Production Economics 165, 2015, pp. 234-246.

[3] Elia, G., G. Polimeno, G. Solazzo, and G. Passiante, “A multi-dimension framework for value creation through Big Data", Industrial Marketing Management 90, 2020, pp. 508-522.

[4] Wiener, M., C. Saunders, and M. Marabelli, "Big-data business models: A critical literature review and multiperspective research framework", Journal of Information Technology 35(1), 2020, pp. 66-91.

[5] Chen, H.-M., R. Kazman, and F. Matthes, "Demystifying Big Data Adoption: Beyond IT Fashion and Relative Advantage", Proceedings of the 20th DIGIT Workshop, (2015).

[6] Chen, H.-M., R. Kazman, R. Schütz, and F. Matthes, "How Lufthansa Capitalized on Big Data for Business Model Renovation", MIS Quarterly Executive 16(1), 2017, pp. 19-34.

[7] Gupta, M., and J.F. George, "Toward the development of a big data analytics capability", Information \& Management 53(8), 2016, pp. 1049-1064.
[8] Troilo, G., L.M. De Luca, and P. Guenzi, "Linking DataRich Environments with Service Innovation in Incumbent Firms: A Conceptual Framework and Research Propositions", Journal of Product Innovation Management 34(5), 2017, pp. 617-639.

[9] Akter, S., S.F. Wamba, A. Gunasekaran, R. Dubey, and S.J. Childe, "How to improve firm performance using big data analytics capability and business strategy alignment?", International Journal of Production Economics 182, 2016, pp. 113-131.

[10] Lehrer, C., A. Wieneke, J. vom Brocke, R. Jung, and S. Seidel, "How Big Data Analytics Enables Service Innovation: Materiality, Affordance, and the Individualization of Service", Journal of Management Information Systems 35(2), 2018, pp. 424-460.

[11] Li, S., G.C. Peng, and F. Xing, "Barriers of embedding big data solutions in smart factories: insights from SAP consultants", Industrial Management \& Data Systems 119(5), 2019, pp. 1147-1164.

[12] Mikalef, P., I.O. Pappas, J. Krogstie, and P.A. Pavlou, "Big data and business analytics: A research agenda for realizing business value", Information \& Management 57(1), 2020, pp. 1-15.

[13] McAfee, A., and E. Brynjolfsson, "Big Data: The Management Revolution", Harvard Business Review 90(10), 2012, pp. 60-68.

[14] Constantiou, I.D., and J. Kallinikos, "New Games, New Rules: Big Data and the Changing Context of Strategy", Journal of Information Technology 30(1), 2015, pp. 4457.

[15] Günther, W.A., M.H. Rezazade Mehrizi, M. Huysman, and F. Feldberg, "Debating big data: A literature review on realizing value from big data", The Journal of Strategic Information Systems 26(3), 2017, pp. 191-209.

[16] Loebbecke, C., and A. Picot, "Reflections on societal and business model transformation arising from digitization and big data analytics: A research agenda", The Journal of Strategic Information Systems 24(3), 2015, pp. 149157.

[17] Grover, V., R.H.L. Chiang, T.-P. Liang, and D. Zhang, "Creating Strategic Business Value from Big Data Analytics: A Research Framework", Journal of Management Information Systems 35(2), 2018, pp. 388423.

[18] Raut, R.D., V.S. Yadav, N. Cheikhrouhou, V.S. Narwane, and B.E. Narkhede, "Big data analytics: Implementation challenges in Indian manufacturing supply chains", Computers in Industry 125, 2021.

[19] Sivarajah, U., M.M. Kamal, Z. Irani, and V. Weerakkody, "Critical analysis of Big Data challenges and analytical methods", Journal of Business Research 70, 2017, pp. 263-286.

[20] Tabesh, P., E. Mousavidin, and S. Hasani, "Implementing big data strategies: A managerial perspective", Business Horizons 62(3), 2019, pp. 347-358.

[21] Alharthi, A., V. Krotov, and M. Bowman, "Addressing barriers to big data", Business Horizons 60(3), 2017, pp. 285-292.

[22] Melville, N., K. Kraemer, and V. Gurbaxani, "Review: Information Technology and Organizational 
Performance: An Integrative Model of IT Business Value", MIS Quarterly 28(2), 2004, pp. 283-322.

[23] Sharma, R., S. Mithas, and A. Kankanhalli, "Transforming decision-making processes: a research agenda for understanding the impact of business analytics on organisations", European Journal of Information Systems 23(4), 2014, pp. 433-441.

[24] Mikalef, P., J. Krogstie, I.O. Pappas, and P. Pavlou, "Exploring the relationship between big data analytics capability and competitive performance: The mediating roles of dynamic and operational capabilities", Information \& Management 57(2), 2020.

[25] Wamba, S., and S. Akter, "Understanding supply chain analytics capabilities and agility for data-rich environments", International Journal of Operations \& Production Management 39(6/7/8), 2019, pp. 887-912.

[26] Kiron, D., P.K. Prentice, and R.B. Ferguson, "The Analytics Mandate", MIT Sloan Management Review 55(2), 2014, pp. 1-21.

[27] vom Brocke, J., A. Simons, B. Niehaves, and K. Reimer, "Reconstructing the Giant: On the Importance of Rigour in Documenting the Literature Search Process", Proceedings of the 17th European Conference on Information Systems, (2009), 2206-2217.

[28] Gioia, D.A., K.G. Corley, and A.L. Hamilton, "Seeking Qualitative Rigor in Inductive Research: Notes on the Gioia Methodology", Organizational Research Methods 16(1), 2013, pp. 15-31.

[29] Strauss, A., and J. Corbin, Basics of qualitative research: Techniques and procedures for developing grounded theory, Sage Publications.

[30] Beath, C.M., and J.W. Ross, "PepsiAmericas: Building an Information Savvy Company", CISR, 2010, pp. 17.

[31] Dremel, C., M.M. Herterich, J. Wulf, and J. vom Brocke, "Actualizing big data analytics affordances: A revelatory case study", Information \& Management 57(1), 2020.

[32] Koch, H., W. Chipidza, and T.R. Kayworth, "Realizing value from shadow analytics: A case study", The Journal of Strategic Information Systems 30(2), 2021, pp. 1-18.

[33] Krishnamoorthi, S., and S.K. Mathew, "Business analytics and business value: A comparative case study", Information \& Management 55(5), 2018, pp. 643-666.

[34] Sejahtera, F., W. Wang, M. Indulska, and S. Sadiq, "Enablers And Inhibitors Of Effective Use Of Big Data: Insights From A Case Study", Proceedings of the 22nd Asia Conference on Information Systems, (2018), 15.

[35] Tim, Y., P. Hallikainen, S.L. Pan, and T. Tamm, "Actualizing business analytics for organizational transformation: A case study of Rovio Entertainment", European Journal of Operational Research 281(3), 2020, pp. 642-655.

[36] Winig, L., "GE's Big Bet on Data and Analytics", MIT Sloan Management Review, 2016, pp. 1-16.

[37] Dai, H.-N., H. Wang, G. Xu, J. Wan, and M. Imran, "Big data analytics for manufacturing internet of things: opportunities, challenges and enabling technologies", Enterprise Information Systems 14(9-10), 2020, pp. 1279-1303.

[38] Khan, M., "Challenges with big data analytics in service supply chains in the UAE", Management Decision 57(8), 2019, pp. 2124-2147.
[39] Kaisler, S., F. Armour, J.A. Espinosa, and W. Money, "Big Data: Issues and Challenges Moving Forward", 2013 46th Hawaii International Conference on System Sciences, IEEE (2013), 995-1004.

[40] Espinosa, J.A., S. Kaisler, F. Armour, and W.H. Money, "Big Data Redux: New Issues and Challenges Moving Forward", Proceedings of the 52nd Hawaii Conference on the System Sciences, (2019).

[41] Kache, F., and S. Seuring, "Challenges and opportunities of digital information at the intersection of Big Data Analytics and supply chain management", International Journal of Operations \& Production Management 37(1), 2017, pp. 10-36.

[42] Koronios, A., J. Gao, and S. Selle, "Big Data Project Success - A Meta Analysis", Proceedings of the 18th Pacific Asia Conference on Information Systems, (2014).

[43] Moktadir, Md.A., S.M. Ali, S.K. Paul, and N. Shukla, "Barriers to big data analytics in manufacturing supply chains: A case study from Bangladesh", Computers \& Industrial Engineering 128, 2019, pp. 1063-1075.

[44] Chen, P.-T., C.-L. Lin, and W.-N. Wu, "Big data management in healthcare: Adoption challenges and implications", International Journal of Information Management 53, 2020.

[45] Staegemann, D., N. Jamous, M. Volk, and K. Turowski, "Understanding Issues in Big Data Applications - A Multidimensional Endeavor", Processings of the 25th Americas Conference on Information Systems, (2019).

[46] Halaweh, M., "Conceptual Model for Successful Implementation of Big Data in Organizations", Journal of International Technology and Information Management 24(2), 2015, pp. 21-34.

[47] Vidgen, R., S. Shaw, and D.B. Grant, "Management challenges in creating value from business analytics", European Journal of Operational Research 261(2), 2017, pp. 626-639.

[48] Dremel, C., "Barriers to the Adoption of Big Data Analytics in the Automotive Sector", Proceedings of the 23rd Americas Conference on Information Systems, (2017).

[49] Jensen, M.H., P.A. Nielsen, and J.S. Persson, "Managing Big Data Analytics Projects: The Challenges of Realizing Value", Proceedings of the 27th European Conference on Information Systems.

[50] Illa, P.K., and N. Padhi, "Practical Guide to Smart Factory Transition Using IoT, Big Data and Edge Analytics", IEEE Access 6, 2018, pp. 55162-55170.

[51] Dietrich, D., B. Heller, and B. Yang, "Data Analytics Lifecycle", In Data Science \& Big Data Analytics: Discoverying, Analyzing, Visualizing, and Presenting Data. John Wiley \& Sons, Inc., Indianapolis, Indiana, 2015, 25-62.

[52] DAMA International, ed., DAMA-DMBOK: Data Management Body of Knowledge, Technics Publications, New Jersey.

[53] Mikalef, P., M. Boura, G. Lekakos, and J. Krogstie, "The role of information governance in big data analytics driven innovation", Information \& Management 57(7), 2020. 